\title{
Generalizations of Dedekind sums and their reciprocity laws
}

by

Yumiko Nagasaka, Kaori Ota and Chizuru Sekine (Tokyo)

1. Introduction. Dedekind sums are defined for positive integers $k, h$ with $(k, h)=1$ by

$$
s(k, h)=\sum_{a=1}^{h-1} \bar{B}_{1}\left(\frac{a}{h}\right) \bar{B}_{1}\left(\frac{k a}{h}\right) \quad \text { and } \quad s(h, k)=\sum_{b=1}^{k-1} \bar{B}_{1}\left(\frac{b}{k}\right) \bar{B}_{1}\left(\frac{h b}{k}\right),
$$

where $\bar{B}_{1}(z)$ is the first Bernoulli function (see Theorem A below). They were originally introduced in connection with the modular properties of the Dedekind $\eta$-function, and Dedekind proved the reciprocity law

$$
s(k, h)+s(h, k)=\frac{1}{12}\left(\frac{k}{h}+\frac{h}{k}+\frac{1}{k h}\right)-\frac{1}{4}
$$

(see [18]). These sums were later generalized by various people (Apostol, Berndt, Carlitz, Hall-Wilson-Zagier, Mikolás, Rademacher, Solomon, Zagier, ...), and the corresponding reciprocity laws were obtained. Then general types of sums such as

$$
\sum_{0 \leq i_{1}, \ldots, i_{n}<a} \bar{B}_{r_{1}}\left(\frac{i_{1}+\lambda_{1}}{a}\right) \ldots \bar{B}_{r_{n}}\left(\frac{i_{n}+\lambda_{n}}{a}\right)
$$

were considered and the corresponding reciprocity laws were proved, where $a$ is a positive integer, $r_{1}, \ldots, r_{n}$ are non-negative integers, and $\lambda_{1}, \ldots, \lambda_{n}$ are real numbers (see $[8,9,11]$ ).

In this paper we focus on the sums considered by Apostol. Apostol's reciprocity law is the following:

TheOrem A (Apostol [1]). For a positive integer $n$, set

$$
s_{n}(h, k)=\sum_{a=1}^{k-1} \frac{a}{k} \bar{B}_{n}\left(\frac{h a}{k}\right) \quad \text { and } \quad s_{n}(k, h)=\sum_{b=1}^{h-1} \frac{b}{h} \bar{B}_{n}\left(\frac{k b}{h}\right),
$$

2000 Mathematics Subject Classification: 11F20, 11M35, 11M41, $11 \mathrm{~B} 68$. 
where $\bar{B}_{n}$ is the nth Bernoulli function, i.e.,

$$
\begin{aligned}
& \bar{B}_{n}(x)=B_{n}(\{x\}) \quad \text { if } n>1 \text {, } \\
& \bar{B}_{1}(x)= \begin{cases}B_{1}(\{x\}) & \text { if } x \notin \mathbb{Z}, \\
0 & \text { if } x \in \mathbb{Z} .\end{cases}
\end{aligned}
$$

Here $B_{n}(*)$ is the nth Bernoulli polynomial, and $\{x\}$ denotes the fractional part of a real number $x$, i.e., $0 \leq\{x\}<1$. Then the following identity holds for $n$ odd:

$$
\frac{1}{n}\left\{k^{n-1} s_{n}(h, k)+h^{n-1} s_{n}(k, h)\right\}=\frac{\left({ }^{1} B h-{ }^{2} B k\right)^{n+1}}{n(n+1) k h}+\frac{B_{n+1}}{(n+1) k h},
$$

where

$$
\left({ }^{1} B h-{ }^{2} B k\right)^{n+1}=\sum_{i=0}^{n+1}\left(\begin{array}{c}
n+1 \\
i
\end{array}\right)(-1)^{n+1-i} B_{i} h^{i} B_{n+1-i} k^{n+1-i} .
$$

We show how to prove Theorem A by using values at non-positive integers of Barnes's double zeta function $\widetilde{\zeta}_{2}(s ;(k, h))$. Although there are simpler proofs of Theorem A without using a zeta function, this is a new proof which makes it possible to obtain new kinds of Dedekind sums and explicit reciprocity laws for them in a unified way. The function $\widetilde{\zeta}_{2}(s ;(k, h))$ is defined by

$$
\widetilde{\zeta}_{2}(s ;(k, h))=\sum_{\substack{m, n=0 \\(m, n) \neq(0,0)}}^{\infty} \frac{1}{(k m+h n)^{s}}
$$

for $\operatorname{Re}(s)>2$, and is analytically continued to the whole complex plane with some poles. The proof goes as follows: From contour integral representation we know that

$$
\widetilde{\zeta}_{2}(1-n ;(k, h))=\frac{{ }_{2} S_{n}^{\prime}(0 ;(k, h))}{n}-\delta=\frac{\left({ }^{1} B k+{ }^{2} B h\right)^{n+1}}{n(n+1) k h}-\delta,
$$

where $\delta=1$ when $n=1$ and $\delta=0$ otherwise, ${ }_{2} S_{n}^{\prime}(0 ;(k, h))$ is the first derivative of the double $n$th Bernoulli polynomial introduced by Barnes, and

$$
\left({ }^{1} B k+{ }^{2} B h\right)^{n+1}=\sum_{j=0}^{n+1}\left(\begin{array}{c}
n+1 \\
j
\end{array}\right) B_{j} k^{j} B_{n+1-j} h^{n+1-j}
$$

(see Section 2). On the other hand, by computing $\widetilde{\zeta}_{2}(1-n ;(k, h))$ in a suitable way, we obtain terms involving $s_{n}(h, k)$ and $s_{n}(k, h)$. Therefore we have two expressions of $\widetilde{\zeta}_{2}(1-n ;(k, h))$, and by equating the two, Apostol's reciprocity law is obtained. 
Following this idea, we consider three kinds of generalizations of Apostol's sums. The first generalization is obtained by using Barnes's double zeta function $\zeta_{2}(s ; \alpha,(k, h))$ with parameters $\alpha$ and $(k, h)$, which is defined by

$$
\zeta_{2}(s ; \alpha,(k, h))=\sum_{m, n=0}^{\infty} \frac{1}{(\alpha+k m+h n)^{s}}
$$

for a complex number $\alpha$ with positive real part, and from the value $\zeta_{2}(1-n$; $\alpha,(k, h))$ we obtain the law for sums

$$
\begin{aligned}
& s_{n}(\alpha ;(k, h))=\sum_{a=1}^{h-1} \frac{a}{h} \bar{B}_{n}\left(\frac{\alpha+k a}{h}\right), \\
& s_{n}(\alpha ;(h, k))=\sum_{b=1}^{k-1} \frac{b}{k} \bar{B}_{n}\left(\frac{\alpha+h b}{k}\right)
\end{aligned}
$$

for real $\alpha$ with $0<\alpha<h+k$. As we mentioned above, these sums have been considered in far more general settings (cf. (1.1)), and the corresponding reciprocity laws were obtained. The second generalization involves Dirichlet characters. Dedekind sums with characters have been considered for $n=1$ by Berndt, and he proved reciprocity laws by using either Eisenstein series with characters (cf. $[4,5]$ ), integrals such as contour integrals and RiemannStieltjes integrals, or the Poisson summation formula (cf. [6]). Here we consider different kinds of sums. For a Dirichlet character $\chi \bmod k h$, we define $\widetilde{\zeta}_{2}(s ;(k, h), \chi)$ by

$$
\widetilde{\zeta}_{2}(s ;(k, h), \chi)=\sum_{\substack{m, n=0 \\(m, n) \neq(0,0)}}^{\infty} \frac{\chi(k m+h n)}{(k m+h n)^{s}} .
$$

Then from $\widetilde{\zeta}_{2}(1-n ;(k, h), \chi)$ we obtain the law for sums

$$
\begin{aligned}
& s_{n}(\chi ;(k, h))=k^{n-1} \sum_{a=0}^{h-1} \sum_{b=0}^{k-1} \frac{a}{h} \chi(k a+h b) \bar{B}_{n}\left(\frac{a}{h}+\frac{b}{k}\right), \\
& s_{n}(\chi ;(h, k))=h^{n-1} \sum_{a=0}^{h-1} \sum_{b=0}^{k-1} \frac{b}{k} \chi(k a+h b) \bar{B}_{n}\left(\frac{a}{h}+\frac{b}{k}\right)
\end{aligned}
$$

(see Theorem 3.6). In particular, when $n=1$ and $\chi=\chi_{1} \chi_{2}$ with $\chi_{1}$ (resp. $\chi_{2}$ ) a character $\bmod k($ resp. $\bmod h), \widetilde{\zeta}_{2}(0 ;(k, h), \chi)$ is expressed in terms of $B_{1, \chi_{i}}$ or $B_{2, \chi_{i}}$, so the law has a simple form (see Corollary 3.7). As an application, we obtain the formulae for class numbers for imaginary quadratic number fields (cf. [17]). The last generalization is given by considering Barnes's $(r+1)$-ple zeta function $\widetilde{\zeta}_{r+1}\left(s ;\left(k, h, z_{1}, \ldots, z_{r-1}\right)\right)$ with param- 
eters $\left(k, h, z_{1}, \ldots, z_{r-1}\right)$, which is defined by

$$
\begin{aligned}
\widetilde{\zeta}_{r+1}\left(s ;\left(k, h, z_{1}, \ldots, z_{r-1}\right)\right) & \\
= & \sum_{\substack{m_{1}, \ldots, m_{r+1}=0 \\
\left(m_{1}, \ldots, m_{r+1} \neq(0, \ldots, 0)\right.}}^{\infty} \frac{1}{\left(m_{1} k+m_{2} h+m_{3} z_{1}+\ldots+m_{r+1} z_{r-1}\right)^{s}},
\end{aligned}
$$

and the value at a non-positive integer $1-n$ is given by the derivative of the $(r+1)$-ple Bernoulli polynomial ${ }_{r+1} S_{n}^{\prime}\left(0 ;\left(k, h, z_{1}, \ldots, z_{r-1}\right)\right)$ introduced by Barnes (see $[2,3]$ or $(2.7))$. The law is for $\left(\mathbf{S}_{r}\right)_{n}\left(h, k ;\left(z_{1}, \ldots, z_{r-1}\right)\right)$ and $\left(\mathbf{S}_{r}\right)_{n}\left(k, h ;\left(z_{1}, \ldots, z_{r-1}\right)\right)$, which we call $r$-ple Dedekind sums, defined by

$$
\begin{aligned}
& \left(\mathbf{S}_{r}\right)_{n}\left(h, k ;\left(z_{1}, \ldots, z_{r-1}\right)\right) \\
& \quad=\sum_{a=1}^{k-1} \frac{a}{k}{ }_{r} S_{n}^{\prime}\left(\left\{\frac{h a}{k}\right\} ;\left(1, \frac{z_{1}}{k}, \ldots, \frac{z_{r-1}}{k}\right)\right),
\end{aligned}
$$

where $z_{1}, \ldots, z_{r-1}$ are non-zero complex numbers. In [11], the sums of type (1.1) are called $n$-ple Dedekind sums. Since (1.1) and (1.3) have different sets of parameters and there seems to be no other suitable name for (1.3), we also call it the $r$-ple Dedekind sum in this paper. Our sums have the merit that the law has a very simple form (see Theorem 4.2). When $r=1$, they coincide with Apostol's sums.

We note that Egami has proved the reciprocity law for (1.1) by using multiple zeta functions associated to a cone (see [9]). We also note that by considering the first derivative of $\widetilde{\zeta}_{2}(s ;(k, h))$ with respect to $s$, we can define "derivatives" of Dedekind sums and prove the reciprocity law for them (see $[16]$ ).

In Section 2 we review Barnes's multiple zeta functions and derivatives of multiple Bernoulli polynomials. In Section 3 we first prove Apostol's reciprocity law by using $\widetilde{\zeta}_{2}(1-n ;(k, h))$. Then the laws for shifted sums $s_{n}(\alpha ;(k, h))$ and for sums with a character $s_{n}(\chi ;(k, h))$ are proved. In the last section we prove the reciprocity law for our $r$-ple Dedekind sums.

\section{Review of Barnes's multiple zeta functions and multiple} Bernoulli polynomials. In this section we give a brief summary for Barnes's multiple zeta functions and multiple Bernoulli polynomials only needed in subsequent sections. For more details, see $[2,3]$.

Definition 2.1. The Bernoulli numbers $B_{n}$ and the Bernoulli polynomials $B_{n}(u)$ are defined by 


$$
\frac{t}{e^{t}-1}=\sum_{n=0}^{\infty} \frac{B_{n}}{n !} t^{n} \quad \text { and } \quad \frac{t e^{u t}}{e^{t}-1}=\sum_{n=0}^{\infty} \frac{B_{n}(u)}{n !} t^{n} .
$$

Also for a Dirichlet character $\chi$ of conductor $f$, we define $B_{n, \chi}$ by

$$
\sum_{a=1}^{f} \frac{\chi(a) t e^{a t}}{e^{f t}-1}=\sum_{n=0}^{\infty} B_{n, \chi} \frac{t^{n}}{n !} .
$$

Let $r$ be a positive integer.

DeFinition 2.2. Let $\alpha, \omega_{1}, \ldots, \omega_{r}$ be complex numbers such that $\omega_{i} \neq 0$ for each $i$, and set $\widetilde{\omega}=\left(\omega_{1}, \ldots, \omega_{r}\right)$. The $r$-ple nth Bernoulli polynomial of $\alpha$ with parameters $\omega_{1}, \ldots, \omega_{r}$ is a polynomial ${ }_{r} S_{n}(\alpha ; \widetilde{\omega})$ which vanishes when $\alpha=0$ and whose first derivative ${ }_{r} S_{n}^{\prime}(\alpha ; \widetilde{\omega})$ appears as the coefficient of $t^{n}$ in the expression

$$
\frac{(-1)^{r} t e^{-\alpha t}}{\prod_{i=1}^{r}\left(1-e^{-\omega_{i} t}\right)}=\sum_{k=1}^{r} \frac{(-1)^{k} A_{k}(\alpha ; \widetilde{\omega})}{t^{k-1}}+\sum_{n=1}^{\infty} \frac{(-1)^{n-1}{ }_{r} S_{n}^{\prime}(\alpha ; \widetilde{\omega})}{n !} t^{n} .
$$

This expression is valid for $t$ with $|t|<\min \left(\left|2 \pi / \omega_{1}\right|, \ldots,\left|2 \pi / \omega_{r}\right|\right)$. Here we can show that $A_{k}(\alpha ; \widetilde{\omega})={ }_{r} S_{1}^{(k+1)}(\alpha ; \widetilde{\omega})$, the $(k+1)$ th derivative of ${ }_{r} S_{1}(\alpha ; \widetilde{\omega})$ with respect to $\alpha$.

REMARK 2.3. (1) ${ }_{1} S_{n}^{\prime}(\alpha ;(\omega)), B_{n}(u)$ and $B_{n}$ are related by

$$
{ }_{1} S_{n}^{\prime}(\alpha ;(\omega))=\omega^{n-1} B_{n}\left(\frac{\alpha}{\omega}\right) \text { and }{ }_{1} S_{n}^{\prime}(0 ;(\omega))=\omega^{n-1} B_{n} \quad \text { for } n \geq 1 .
$$

This is easily seen by the definition of ${ }_{1} S_{n}^{\prime}$.

(2) For a complex number $\lambda \neq 0$,

$$
{ }_{r} S_{n}^{\prime}(\alpha ; \widetilde{\omega})=\lambda^{1-n} S_{n}^{\prime}(\lambda \alpha ; \lambda \widetilde{\omega}) .
$$

This is clear from the generating function.

(3) We have the following expression for ${ }_{r} S_{n}^{\prime}(\alpha ; \widetilde{\omega})$ :

$$
{ }_{r} S_{n}^{\prime}(\alpha ; \widetilde{\omega})=\frac{\left({ }^{1} B \omega_{1}+\ldots+{ }^{r} B \omega_{r}+\alpha\right)^{n+r-1} n !}{\prod_{i=1}^{r} \omega_{i} \cdot(n+r-1) !},
$$

where in the multinomial expansion of $\left({ }^{1} B \omega_{1}+\ldots+{ }^{r} B \omega_{r}+\alpha\right)^{N}$ in (2.4) we mean that

$\left({ }^{i} B\right)^{j}$ (the $j$ th power of $\left.{ }^{i} B\right)=B_{j} \quad$ but $\quad\left({ }^{i} B\right)^{j} \cdot\left({ }^{l} B\right)^{k} \neq B_{j+k} \quad$ if $i \neq l$.

This is shown by the following identities using (2.1): 


$$
\begin{aligned}
& \frac{(-1)^{r} t e^{-\alpha t}}{\prod_{i=1}^{r}\left(1-e^{-\omega_{i} t}\right)} \\
& \quad=\prod_{i=1}^{r} \frac{\left(-\omega_{i} t\right)}{e^{-\omega_{i} t}-1} \cdot e^{-\alpha t} \cdot \frac{1}{t^{r-1}} \cdot \frac{(-1)^{r}}{\prod_{i=1}^{r} \omega_{i}} \\
& \quad=\prod_{i=1}^{r}\left(\sum_{n_{i}=0}^{\infty} \frac{B_{n_{i}}}{n_{i} !}\left(-\omega_{i} t\right)^{n_{i}}\right) \cdot\left(\sum_{n=0}^{\infty} \frac{1}{n !}(-\alpha t)^{n}\right) \cdot \frac{1}{t^{r-1}} \cdot \frac{(-1)^{r}}{\prod_{i=1}^{r} \omega_{i}} \\
& \quad=\sum_{N=0}^{\infty} \frac{\left({ }^{1} B \omega_{1}+\ldots+{ }^{r} B \omega_{r}+\alpha\right)^{N}(-t)^{N-(r-1)}}{N !} \cdot \frac{-1}{\prod_{i=1}^{r} \omega_{i}} .
\end{aligned}
$$

Although ${ }_{r} S_{n}(\alpha ; \widetilde{\omega})$ is called the $r$-ple Bernoulli polynomial, it is its first derivative ${ }_{r} S_{n}^{\prime}(\alpha ; \widetilde{\omega})$ which gives the value of the $r$-ple zeta function.

Definition 2.4. Let $\alpha, \omega_{1}, \ldots, \omega_{r}$ be complex numbers with positive real parts, and set $\widetilde{\omega}=\left(\omega_{1}, \ldots, \omega_{r}\right)$. Barnes's $r$-ple zeta function $\zeta_{r}(s ; \alpha, \widetilde{\omega})$ with parameters $\alpha$ and $\widetilde{\omega}$ is defined by

$$
\zeta_{r}(s ; \alpha, \widetilde{\omega})=\sum_{m_{1}, \ldots, m_{r}=0}^{\infty} \frac{1}{\left(\alpha+\omega_{1} m_{1}+\ldots+\omega_{r} m_{r}\right)^{s}} \quad \text { for } \operatorname{Re}(s)>r .
$$

Here $u^{s}=\exp (s \log u)$ and $\log u=\log |u|+i \arg u$ with $-\pi<\arg u<\pi$ for any complex number $u$ not on the non-positive real axis. We note that when $r=1$,

$$
\zeta_{1}\left(s ; \alpha,\left(\omega_{1}\right)\right)=\sum_{m_{1}=0}^{\infty} \frac{1}{\left(\alpha+\omega_{1} m_{1}\right)^{s}}=\frac{1}{\omega_{1}^{s}} \zeta\left(s, \frac{\alpha}{\omega_{1}}\right),
$$

where $\zeta\left(s, \alpha / \omega_{1}\right)$ is the Hurwitz zeta function.

Analytic continuation and special values are given by the contour integral representation of $\zeta_{r}(s ; \alpha, \widetilde{\omega})$ as in the following theorem (cf. [2,3]).

THEOREM 2.5. $\zeta_{r}$ is expressed as a contour integral:

$$
\zeta_{r}(s ; \alpha, \widetilde{\omega})=\frac{\Gamma(1-s) e^{-s \pi i}}{2 \pi i} \int_{I(\lambda, \infty)} \frac{e^{-\alpha t} t^{s-1}}{\prod_{i=1}^{r}\left(1-e^{-\omega_{i} t}\right)} d t,
$$

where $0<\lambda<\min \left(\left|2 \pi / \omega_{1}\right|, \ldots,\left|2 \pi / \omega_{r}\right|\right)$ and $I_{(\lambda, \infty)}$ is the path from $+\infty$ to $\lambda$ along the real axis, going along the circle around 0 of radius $\lambda$ counterclockwise to $\lambda$, and then going back to $+\infty$. This expression gives us the analytic continuation of $\zeta_{r}$ to the whole complex plane, and also for a positive integer $n$ we have

$$
\begin{aligned}
\zeta_{r}(1-n ; \alpha, \widetilde{\omega}) & =\frac{(-1)^{r}{ }_{r} S_{n}^{\prime}(\alpha ; \widetilde{\omega})}{n} \\
& =\frac{(-1)^{r}\left({ }^{1} B \omega_{1}+\ldots+{ }^{r} B \omega_{r}+\alpha\right)^{n+r-1}}{n(n+1) \ldots(n+r-1) \cdot \prod_{i=1}^{r} \omega_{i}} .
\end{aligned}
$$


When $r=1$, this is well known as the value of the Hurwitz zeta function:

$$
\zeta(1-n, \alpha)=-\frac{B_{n}(\alpha)}{n} .
$$

Definition 2.6. Let $\widetilde{\omega}=\left(\omega_{1}, \ldots, \omega_{r}\right)$ be as in Definition 2.4. We define Barnes's r-ple zeta function $\widetilde{\zeta}_{r}(s ; \widetilde{\omega})$ with parameters $\widetilde{\omega}$ by

$$
\widetilde{\zeta}_{r}(s ; \widetilde{\omega})=\sum_{\substack{m_{1}, \ldots, m_{r}=0 \\\left(m_{1}, \ldots, m_{r}\right) \neq(0, \ldots, 0)}}^{\infty} \frac{1}{\left(\omega_{1} m_{1}+\ldots+\omega_{r} m_{r}\right)^{s}} .
$$

This series is a replacement of $\zeta_{r}$ for $\alpha=0$, and when $r=1$,

$$
\widetilde{\zeta}_{1}\left(s ;\left(\omega_{1}\right)\right)=\sum_{m=1}^{\infty} \frac{1}{\left(\omega_{1} m\right)^{s}}=\frac{1}{\omega_{1}^{s}} \zeta(s),
$$

where $\zeta(s)$ is the Riemann zeta function. The function $\widetilde{\zeta}_{r}(s ; \widetilde{\omega})$ is also continued analytically to the whole complex plane, and

$$
\widetilde{\zeta}_{r}(1-n ; \widetilde{\omega})=\frac{(-1)^{r}{ }_{r} S_{n}^{\prime}(0 ; \widetilde{\omega})}{n}-\delta,
$$

where $\delta=1$ for $n=1$ and $\delta=0$ otherwise. This can be shown for example by induction using the identity

$$
\widetilde{\zeta}_{r}(s ; \widetilde{\omega})=\zeta_{r}\left(s ; \omega_{1}, \widetilde{\omega}\right)+\widetilde{\zeta}_{r-1}\left(s ;\left(\omega_{2}, \ldots, \omega_{r}\right)\right) .
$$

We note that

$$
\zeta(1-n)=\zeta(1-n, 1)=-\frac{B_{n}}{n}-\delta .
$$

In subsequent sections we need to treat $\zeta(s)$ and $\zeta(s, \alpha)$, and also $\zeta_{r}$ and $\widetilde{\zeta}_{r}$, simultaneously, so we use the following notation:

$$
\begin{aligned}
\zeta^{*}(s, \alpha) & = \begin{cases}\zeta(s, \alpha) & \text { if } \operatorname{Re}(\alpha)>0, \\
\zeta(s) & \text { if } \alpha=0 ;\end{cases} \\
\zeta_{r}^{*}(s ; \alpha, \widetilde{\omega}) & = \begin{cases}\zeta_{r}(s ; \alpha, \widetilde{\omega}) & \text { if } \operatorname{Re}(\alpha)>0, \\
\widetilde{\zeta}_{r}(s ; \widetilde{\omega}) & \text { if } \alpha=0 .\end{cases}
\end{aligned}
$$

3. Reciprocity laws for simple Dedekind sums. In this section we prove the reciprocity law for Apostol sums (Theorem A in Section 1) and for shifted Dedekind sums by using the double zeta function. Then from the double zeta function with a Dirichlet character, we define Dedekind sums with a character and prove the corresponding reciprocity law. Throughout this section and the next, $k$ and $h$ are relatively prime positive integers.

First we show that Theorem A is equivalent to the following Theorem 3.1 when $n$ is odd. 
THEOREM 3.1. For any positive integer n, we have $\widetilde{\zeta}_{2}(1-n ;(k, h))=\frac{1}{n}\left\{h^{n-1} s_{n}(k, h)+k^{n-1} s_{n}(h, k)\right\}-\frac{1}{h k} \cdot \frac{B_{n+1}}{n+1}-\frac{B_{n}}{n}-\delta$, where $\delta=1$ when $n=1$ and $\delta=0$ otherwise.

Proof of the equivalence when $n$ is odd. It suffices to show that for $n$ odd

$$
\widetilde{\zeta}_{2}(1-n ;(k, h))=\frac{\left({ }^{1} B k-{ }^{2} B h\right)^{n+1}}{n(n+1) k h}-\frac{B_{n}}{n}-\delta .
$$

The identity

$$
\frac{t}{\left(1-e^{-k t}\right)\left(1-e^{-h t}\right)}=\frac{t}{1-e^{-k t}}-\frac{t}{\left(1-e^{-k t}\right)\left(1-e^{h t}\right)}
$$

implies

$$
{ }_{2} S_{n}^{\prime}(0 ;(k, h))=-k^{n-1} B_{n}-{ }_{2} S_{n}^{\prime}(0 ;(k,-h)) .
$$

When $n$ is odd with $n \geq 3, B_{n}=0$, so ${ }_{2} S_{n}^{\prime}(0 ;(k, h))=-B_{n}-{ }_{2} S_{n}^{\prime}(0 ;(k,-h))$ for $n$ odd with $n \geq 1$. Then from (2.4) and (2.7), we obtain (3.1).

Now we give a proof of Theorem 3.1.

Proof of Theorem 3.1. Firstly we deform $\widetilde{\zeta}_{2}(s ;(k, h))$. We have

$$
\begin{aligned}
\widetilde{\zeta}_{2}(s ;(k, h)) & =\sum_{\substack{m, n=0 \\
(m, n) \neq(0,0)}}^{\infty} \frac{1}{(k m+h n)^{s}} \\
& =\sum_{a=0}^{k-1} \sum_{b=0}^{h-1} \sum_{n^{\prime}, m^{\prime}=0}^{\infty} \frac{1}{\left(h a+k b+h k\left(m^{\prime}+n^{\prime}\right)\right)^{s}}
\end{aligned}
$$

by setting $m=b+h m^{\prime}$ and $n=a+k n^{\prime}$ and varying $a$ from 0 to $k-1$ and $b$ from 0 to $h-1$, respectively. Here $\sum^{\prime \prime}$ means that we sum over all pairs of non-negative integers $\left(m^{\prime}, n^{\prime}\right)$ except for $\left(m^{\prime}, n^{\prime}\right)=(0,0)$ when $a=b=0$. Set $m^{\prime}+n^{\prime}=N$; then

$$
\begin{aligned}
\widetilde{\zeta}_{2}(s ;(k, h)) & =\sum_{a=0}^{k-1} \sum_{b=0}^{h-1} \sum_{N=0}^{\infty} \frac{N+1}{(h a+k b+h k N)^{s}} \\
& =\sum_{a=0}^{k-1} \sum_{b=0}^{h-1} \sum_{N=0}^{\prime} \frac{1}{(h k)^{s}} \cdot \frac{N+1}{\left(\frac{h a+k b}{k h}+N\right)^{s}},
\end{aligned}
$$

where $\sum^{\prime}$ means that we sum over all non-negative integers $N$ except for $N=0$ when $a=b=0$. Hence we have 


$$
\begin{aligned}
\widetilde{\zeta}_{2} & (s ;(k, h)) \\
= & \frac{1}{(h k)^{s}} \sum_{a=0}^{k-1} \sum_{b=0}^{h-1} \sum_{N=0}^{\infty}\left\{\frac{1}{\left(\frac{h a+k b}{h k}+N\right)^{s-1}}+\left(1-\frac{a}{k}-\frac{b}{h}\right) \frac{1}{\left(\frac{h a+k b}{h k}+N\right)^{s}}\right\} \\
= & \frac{1}{(h k)^{s}} \sum_{a=0}^{k-1} \sum_{b=0}^{h-1}\left\{\zeta^{*}\left(s-1, \frac{h a+k b}{h k}\right)+\left(1-\frac{a}{k}-\frac{b}{h}\right) \zeta^{*}\left(s, \frac{h a+k b}{h k}\right)\right\}
\end{aligned}
$$

where $\zeta^{*}(s, \alpha)$ is the function defined in $(2.9)$.

Put $s=1-n$ into the equation, and use (2.6) and (2.8). Then

$$
\begin{aligned}
\widetilde{\zeta}_{2}(1-n ;(k, h)) & \\
= & -\frac{(h k)^{n-1}}{n+1} \sum_{a=0}^{k-1} \sum_{b=0}^{h-1} B_{n+1}\left(\frac{h a+k b}{h k}\right) \\
& -\frac{(h k)^{n-1}}{n} \sum_{a=0}^{k-1} \sum_{b=0}^{h-1}\left(1-\frac{a}{k}-\frac{b}{h}\right) B_{n}\left(\frac{h a+k b}{h k}\right)-\delta .
\end{aligned}
$$

In order to compute sums on the right hand side of (3.2), we need the following lemma.

Lemma 3.2. (1) The Bernoulli polynomials $B_{n}(x)$ satisfy the difference equation

$$
B_{n}(x+1)-B_{n}(x)=n x^{n-1} \quad \text { if } n \geq 1 .
$$

(2) For real $x$ and a positive integer $N$, we have

$$
\sum_{i=0}^{N-1} \bar{B}_{n}\left(x+\frac{i}{N}\right)=\frac{\bar{B}_{n}(N x)}{N^{n-1}} .
$$

In particular,

$$
\sum_{i=0}^{N-1} \bar{B}_{n}\left(\frac{i}{N}\right)=\frac{B_{n}}{N^{n-1}}+\eta,
$$

where $\eta=1 / 2$ when $n=1$ and $\eta=0$ otherwise.

(3) Let $\chi$ be a Dirichlet character of conductor $f$. Then for any multiple $F$ of $f$,

$$
B_{n, \chi}=F^{n-1} \sum_{a=1}^{F} \chi(a) B_{n}\left(\frac{a}{F}\right) .
$$

Proof. (1) and (3) can be easily shown from the generating functions.

As for (2), since both sides have period $1 / N$, it suffices to prove it for $0 \leq x<1 / N$. But then it is well known (cf. [15]). 
Now we go back to the identity (3.2). For the values of $a$ and $b$ in our sums of (3.2) we have

Also we note that

$$
0 \leq \frac{h a+k b}{h k}=\frac{a}{k}+\frac{b}{h}<2 \text { and } \frac{h a+k b}{h k} \neq 1 .
$$

$$
\left\{\left\{\frac{h a+k b}{h k}\right\} \mid a, b \in \mathbb{Z}, 0 \leq a<k, 0 \leq b<h\right\}=\left\{\frac{i}{h k} \mid 0 \leq i \leq h k-1\right\} .
$$

Let $S$ denote the set

$$
S=\left\{(a, b) \in \mathbb{Z}^{2} \mid 0 \leq a<k, 0 \leq b<h, a / k+b / h>1\right\} .
$$

Then sums in (3.2) can be simplified by using Lemma 3.2(1), (2). For example we obtain the following identity:

$$
\begin{aligned}
& \sum_{a=0}^{k-1} \sum_{b=0}^{h-1}\left(1-\frac{a}{k}-\frac{b}{h}\right) B_{n}\left(\frac{h a+k b}{h k}\right) \\
& =\sum_{a=0}^{k-1} \sum_{b=0}^{h-1}\left(1-\frac{a}{k}-\frac{b}{h}\right) \bar{B}_{n}\left(\frac{h a+k b}{h k}\right)-n \sum_{(a, b) \in S}\left(\frac{a}{k}+\frac{b}{h}-1\right)^{n}+\mu \\
& =\frac{B_{n}}{(h k)^{n-1}}-\frac{1}{k^{n-1}} \sum_{b=0}^{h-1} \frac{b}{h} \bar{B}_{n}\left(\frac{k b}{h}\right) \\
& \quad-\frac{1}{h^{n-1}} \sum_{a=0}^{k-1} \frac{a}{k} \bar{B}_{n}\left(\frac{h a}{k}\right)-n \sum_{(a, b) \in S}\left(\frac{a}{k}+\frac{b}{h}-1\right)^{n},
\end{aligned}
$$

where $\mu=-1 / 2$ when $n=1$ and $\mu=0$ otherwise. This $\mu$ comes from $B_{1}(0)=\bar{B}_{1}(0)-1 / 2$.

Thus

$$
\begin{aligned}
\widetilde{\zeta}_{2}(1- & n ;(k, h)) \\
= & -\frac{(h k)^{n-1}}{n+1}\left\{\frac{B_{n+1}}{(h k)^{n}}+(n+1) \sum_{(a, b) \in S}\left(\frac{a}{k}+\frac{b}{h}-1\right)^{n}\right\} \\
& -\frac{(h k)^{n-1}}{n}\left\{\frac{B_{n}}{(h k)^{n-1}}-\frac{1}{k^{n-1}} \sum_{b=0}^{h-1} \frac{b}{h} \bar{B}_{n}\left(\frac{k b}{h}\right)\right. \\
& \left.-\frac{1}{h^{n-1}} \sum_{a=0}^{k-1} \frac{a}{k} \bar{B}_{n}\left(\frac{h a}{k}\right)-n \sum_{(a, b) \in S}\left(\frac{a}{k}+\frac{b}{h}-1\right)^{n}\right\}-\delta \\
= & -\frac{1}{n+1} \cdot \frac{B_{n+1}}{h k}-\frac{B_{n}}{n}+\frac{1}{n}\left\{h^{n-1} s_{n}(k, h)+k^{n-1} s_{n}(h, k)\right\}-\delta .
\end{aligned}
$$

This completes the proof of Theorem 3.1. 
Next we consider shifted Dedekind sums.

Definition 3.3. Let $\alpha$ be a real number. We define shifted Dedekind sums $s_{n}(\alpha ;(h, k))$ by

$$
s_{n}(\alpha ;(h, k))=\sum_{c=0}^{k-1} \frac{c}{k} \bar{B}_{n}\left(\frac{\alpha+h c}{k}\right) .
$$

These sums have been considered in far more general settings, and the relevant reciprocity laws were obtained (see Section 1 ).

TheOREM 3.4. Let $\alpha$ be a real number such that $0<\alpha<h+k$. Then for any positive integer $n$,

$$
\begin{aligned}
\frac{1}{n}\left\{h^{n-1}\right. & \left.s_{n}(\alpha ;(k, h))+k^{n-1} s_{n}(\alpha ;(h, k))\right\} \\
& =\frac{1}{h k} \cdot \frac{\bar{B}_{n+1}(\alpha)}{n+1}+\left(1-\frac{\alpha}{h k}\right) \frac{\bar{B}_{n}(\alpha)}{n}+\frac{\left({ }^{1} B h+{ }^{2} B k+\alpha\right)^{n+1}}{n(n+1) h k} .
\end{aligned}
$$

Proof. First we note that by (2.5),

$$
\frac{\left({ }^{1} B h+{ }^{2} B k+\alpha\right){ }^{n+1}}{n(n+1) h k}=\frac{{ }_{2} S_{n}^{\prime}(\alpha ;(k, h))}{n}=\zeta_{2}(1-n ; \alpha,(k, h)) .
$$

Similarly to the proof of Thorem 3.1, we derive

$$
\begin{aligned}
\zeta_{2}(s ; \alpha,(k, h))= & \frac{1}{(h k)^{s}} \sum_{c=0}^{k-1} \sum_{d=0}^{h-1}\left\{\zeta\left(s-1, \frac{h c+k d+\alpha}{h k}\right)\right. \\
& \left.+\left(1-\frac{\alpha}{h k}-\frac{c}{k}-\frac{d}{h}\right) \zeta\left(s, \frac{h c+k d+\alpha}{h k}\right)\right\} .
\end{aligned}
$$

Now put $s=1-n$ into the equation. By (2.6),

$$
\begin{aligned}
\zeta_{2}(1-n ; \alpha,(k, h)) & \\
= & -\frac{(h k)^{n-1}}{n+1} \sum_{c=0}^{k-1} \sum_{d=0}^{h-1} B_{n+1}\left(\frac{h c+k d+\alpha}{h k}\right) \\
& -\frac{(h k)^{n-1}}{n} \sum_{c=0}^{k-1} \sum_{d=0}^{h-1}\left(1-\frac{\alpha}{h k}-\frac{c}{k}-\frac{d}{h}\right) B_{n}\left(\frac{h c+k d+\alpha}{h k}\right) .
\end{aligned}
$$

For the values of $c, d$ and $\alpha$ in the above sums we have

$$
0<\frac{h c+k d+\alpha}{h k}=\frac{c}{k}+\frac{d}{h}+\frac{\alpha}{h k}<2,
$$

and we may have $(h c+k d+\alpha) /(h k)=1$ for some $c, d$. Let $S^{\prime}$ denote the set

$$
S^{\prime}=\left\{(c, d) \in \mathbb{Z}^{2} \mid 0 \leq c<k, 0 \leq d<h, \frac{c}{k}+\frac{d}{h}+\frac{\alpha}{h k} \geq 1\right\} .
$$


We note that

Then

$$
\begin{aligned}
\left\{\left\{\frac{\alpha}{h k}+\frac{h c+k d}{h k}\right\} \mid c, d \in \mathbb{Z}, 0 \leq\right. & c<k, 0 \leq d<h\} \\
& =\left\{\left\{\frac{\alpha}{h k}+\frac{i}{h k}\right\} \mid 0 \leq i \leq h k-1\right\} .
\end{aligned}
$$

$$
\begin{aligned}
\zeta_{2}(1-n ; \alpha,(k, h))= & -\frac{(h k)^{n-1}}{n+1}\left\{\sum_{c=0}^{k-1} \sum_{d=0}^{h-1} \bar{B}_{n+1}\left(\frac{h c+k d+\alpha}{h k}\right)\right. \\
& \left.+(n+1) \sum_{(c, d) \in S^{\prime}}\left(\frac{h c+k d+\alpha}{h k}-1\right)^{n}\right\} \\
& -\frac{(h k)^{n-1}}{n}\left\{\sum_{c=0}^{k-1} \sum_{d=0}^{h-1}\left(1-\frac{\alpha}{h k}-\frac{c}{k}-\frac{d}{h}\right)\right. \\
& \left.\times \bar{B}_{n}\left(\frac{h c+k d+\alpha}{h k}\right)-n \sum_{(c, d) \in S^{\prime}}\left(\frac{h c+k d+\alpha}{h k}-1\right)^{n}\right\} \\
= & -\frac{1}{h k} \cdot \frac{\bar{B}_{n+1}(\alpha)}{n+1}-\left(1-\frac{\alpha}{h k} \frac{\bar{B}_{n}(\alpha)}{n}\right. \\
& +\frac{1}{n}\left\{h^{n-1} s_{n}(\alpha ;(k, h))+k^{n-1} s_{n}(\alpha ;(h, k))\right\} . \text { - }
\end{aligned}
$$

Next we consider Dedekind sums with a character.

Definition 3.5. Let $\chi$ be a Dirichlet character defined mod $l$ with $l \mid h k$. The double zeta function $\widetilde{\zeta}_{2}(s ;(k, h), \chi)$ with parameters $(k, h)$ and $\chi$ is defined by

$$
\widetilde{\zeta}_{2}(s ;(k, h), \chi)=\sum_{\substack{n, m=0 \\(m, n) \neq(0,0)}}^{\infty} \frac{\chi(k m+h n)}{(k m+h n)^{s}} .
$$

From the following identities, $\widetilde{\zeta}_{2}(s ;(k, h), \chi)$ can be analytically continued to the whole complex plane:

$$
\begin{aligned}
\widetilde{\zeta}_{2}(s ;(k, h), \chi) & =\sum_{a=0}^{k-1} \sum_{b=0}^{h-1} \sum_{n^{\prime}, m^{\prime}=0}^{\infty} \frac{\chi\left(h\left(a+k n^{\prime}\right)+k\left(b+h m^{\prime}\right)\right)}{\left(k b+h a+h k\left(m^{\prime}+n^{\prime}\right)\right)^{s}} \\
& =\sum_{a=0}^{k-1} \sum_{b=0}^{h-1} \chi(h a+k b) \zeta_{2}^{*}(s ; h a+k b,(h k, h k)) .
\end{aligned}
$$

Also the Dedekind sum $s_{n}(\chi ;(h, k))$ with a character $\chi$ is defined by

$$
s_{n}(\chi ;(k, h))=k^{n-1} \sum_{a=0}^{k-1} \sum_{b=0}^{h-1} \frac{b}{h} \chi(h a+k b) \bar{B}_{n}\left(\frac{a}{k}+\frac{b}{h}\right) \text {. }
$$


We note that for the principal character $\chi_{0}$,

$$
s_{n}\left(\chi_{0} ;(k, h)\right)=s_{n}(k, h)
$$

from Lemma 3.2(2).

The following reciprocity law holds:

TheOREM 3.6. For any positive integer $n$ and $\chi \neq \chi_{0}$,

$$
\begin{aligned}
& \frac{1}{n}\left\{h^{n-1} s_{n}(\chi ;(k, h))+k^{n-1} s_{n}(\chi ;(h, k))\right\} \\
& =\sum_{a=0}^{k-1} \sum_{b=0}^{h-1} \frac{\chi(h a+k b)\left({ }^{1} B h k+{ }^{2} B h k+h a+k b\right)^{n+1}}{n(n+1)(h k)^{2}}+\frac{1}{h k} \cdot \frac{B_{n+1, \chi}}{n+1}+\frac{B_{n, \chi}}{n} .
\end{aligned}
$$

Proof. We put $s=1-n$ in (3.4) and use (2.5). Then since $\chi(0)=0$, we have

$$
\begin{aligned}
\widetilde{\zeta}_{2}(1-n ;(k, h), \chi) & =\sum_{a=0}^{k-1} \sum_{b=0}^{h-1} \chi(h a+k b) \frac{2 S_{n}^{\prime}(h a+k b ;(h k, h k))}{n} \\
& =\sum_{a=0}^{k-1} \sum_{b=0}^{h-1} \frac{\chi(h a+k b)\left({ }^{1} B h k+{ }^{2} B h k+h a+k b\right)^{n+1}}{n(n+1)(h k)^{2}} .
\end{aligned}
$$

On the other hand, by setting $m^{\prime}+n^{\prime}=N$ in (3.4) we have

$$
\begin{aligned}
\widetilde{\zeta}_{2}(s ;(k, h), \chi)= & \frac{1}{(h k)^{s}} \sum_{a=0}^{k-1} \sum_{b=0}^{h-1} \chi(h a+k b)\left\{\zeta^{*}\left(s-1, \frac{h a+k b}{h k}\right)\right. \\
& \left.+\left(1-\frac{a}{k}-\frac{b}{h}\right) \zeta^{*}\left(s, \frac{h a+k b}{h k}\right)\right\} .
\end{aligned}
$$

Put $s=1-n$ into the previous equality. Then

$$
\begin{aligned}
\widetilde{\zeta}_{2}(1-n ;(k, h), \chi) & =-\frac{(h k)^{n-1}}{n+1} \sum_{a=0}^{k-1} \sum_{b=0}^{h-1} \chi(h a+k b) B_{n+1}\left(\frac{h a+k b}{h k}\right) \\
& -\frac{(h k)^{n-1}}{n} \sum_{a=0}^{k-1} \sum_{b=0}^{h-1} \chi(h a+k b)\left(1-\frac{a}{k}-\frac{b}{h}\right) B_{n}\left(\frac{h a+k b}{h k}\right) \\
= & -\frac{(h k)^{n-1}}{n+1}\left\{\sum_{a=0}^{k-1} \sum_{b=0}^{h-1} \chi(h a+k b) \bar{B}_{n+1}\left(\frac{h a+k b}{h k}\right)\right. \\
& \left.+(n+1) \sum_{(a, b) \in S} \chi(h a+k b)\left(\frac{h a+k b}{h k}-1\right)^{n}\right\}
\end{aligned}
$$




$$
\begin{aligned}
& -\frac{(h k)^{n-1}}{n}\left\{\sum_{a=0}^{k-1} \sum_{b=0}^{h-1} \chi(h a+k b)\left(1-\frac{a}{k}-\frac{b}{h}\right) \bar{B}_{n}\left(\frac{h a+k b}{h k}\right)\right. \\
& \left.-n \sum_{(a, b) \in S} \chi(h a+k b)\left(\frac{a}{k}+\frac{b}{h}-1\right)^{n}\right\},
\end{aligned}
$$

where $S$ is the set in (3.3). From Lemma 3.2(3) we have

$$
\sum_{a=0}^{k-1} \sum_{b=0}^{h-1} \chi(h a+k b) \bar{B}_{n}\left(\frac{h a+k b}{h k}\right)=\frac{B_{n, \chi}}{(h k)^{n-1}} .
$$

So we obtain

$$
\begin{aligned}
\widetilde{\zeta}_{2}(1- & n ;(k, h), \chi) \\
& =-\frac{1}{h k} \cdot \frac{B_{n+1, \chi}}{n+1}-\frac{B_{n, \chi}}{n}+\frac{1}{n}\left\{h^{n-1} s_{n}(\chi,(k, h))+k^{n-1} s_{n}(\chi,(h, k))\right\},
\end{aligned}
$$

which together with (3.5) completes the proof.

As a special case, take $\chi=\chi_{1} \chi_{2}$, where $\chi_{1}$ and $\chi_{2}$ are characters defined $\bmod k$ and $h$, respectively. We define

$$
s_{n}\left(\left(\chi_{1}, k\right),\left(\chi_{2}, h\right)\right)=k^{n-1} \sum_{a=0}^{k-1} \sum_{b=0}^{h-1} \frac{b}{h} \chi_{1}(a) \chi_{2}(b) \bar{B}_{n}\left(\frac{a}{k}+\frac{b}{h}\right) .
$$

Then if we put $u=\chi_{1}(h) \chi_{2}(k)$, we have

$$
s_{n}\left(\chi_{1} \chi_{2},(k, h)\right)=u \cdot s_{n}\left(\left(\chi_{1}, k\right),\left(\chi_{2}, h\right)\right) .
$$

When $n=1$, Theorem 3.6 is simplified as follows: From (3.5),

$\widetilde{\zeta}_{2}\left(0 ;(k, h), \chi_{1} \chi_{2}\right)=u \sum_{a=0}^{k-1} \sum_{b=0}^{h-1} \chi_{1}(a) \chi_{2}(b)\left\{\frac{5}{12}+\frac{b^{2}}{2 h^{2}}+\frac{a^{2}}{2 k^{2}}+\frac{a b}{k h}-\frac{b}{h}-\frac{a}{k}\right\}$.

Now

$$
\begin{aligned}
B_{1, \chi_{1}} & =\sum_{a=0}^{k-1} \chi_{1}(a) B_{1}\left(\frac{a}{k}\right)=\sum_{a=0}^{k-1} \chi_{1}(a)\left(\frac{a}{k}-\frac{1}{2}\right) \\
& = \begin{cases}\sum_{a=0}^{k-1} \frac{a}{k} \chi_{1}(a)-\frac{1}{2} \varphi(k) & \text { if } \chi_{1}=\varphi_{k}, \\
\sum_{a=0}^{k-1} \frac{a}{k} \chi_{1}(a) & \text { if } \chi_{1} \neq \varphi_{k},\end{cases}
\end{aligned}
$$

where $\varphi_{k}$ is the trivial character $\bmod k$ and $\varphi$ is the Euler $\varphi$ function. 
Since we have $B_{1, \chi_{1}}=0$ when $\chi_{1}=\varphi_{k}$,

$$
\sum_{a=0}^{k-1} \frac{a}{k} \chi_{1}(a)= \begin{cases}\frac{1}{2} \varphi(k) & \text { if } \chi_{1}=\varphi_{k} \\ B_{1, \chi_{1}} & \text { if } \chi_{1} \neq \varphi_{k}\end{cases}
$$

Similarly

$$
B_{2, \chi_{1}}=k \sum_{a=0}^{k-1} \chi_{1}(a) B_{2}\left(\frac{a}{k}\right)= \begin{cases}\sum_{a=0}^{k-1} \frac{a^{2} \chi_{1}(a)}{k}-\frac{k}{3} \varphi(k) & \text { if } \chi_{1}=\varphi_{k}, \\ \sum_{a=0}^{k-1} \frac{a^{2} \chi_{1}(a)}{k}-k B_{1, \chi_{1}} & \text { if } \chi_{1} \neq \varphi_{k}\end{cases}
$$

So

$$
\sum_{a=0}^{k-1} \frac{a^{2}}{k} \chi_{1}(a)= \begin{cases}B_{2, \chi_{1}}+\frac{k}{3} \varphi(k) & \text { if } \chi_{1}=\varphi_{k} \\ B_{2, \chi_{1}}+k B_{1, \chi_{1}} & \text { if } \chi_{1} \neq \varphi_{k}\end{cases}
$$

Thus $\widetilde{\zeta}_{2}\left(0 ;(k, h), \chi_{1} \chi_{2}\right)=u \Lambda$, where

$$
\Lambda= \begin{cases}B_{1, \chi_{1}} B_{1, \chi_{2}} & \text { if } \chi_{1} \neq \varphi_{k} \text { and } \chi_{2} \neq \varphi_{h}, \\ \frac{\varphi(h)}{2 k} B_{2, \chi_{1}} & \text { if } \chi_{1} \neq \varphi_{k} \text { and } \chi_{2}=\varphi_{h} \\ \frac{\varphi(k) B_{2, \chi_{2}}}{2 h}+\frac{\varphi(h) B_{2, \chi_{1}}}{2 k} & \text { if } \chi_{1}=\varphi_{k} \text { and } \chi_{2}=\varphi_{h} .\end{cases}
$$

Therefore we have the following corollary.

COROLlary 3.7. Let $\chi_{1}$ and $\chi_{2}$ be characters defined $\bmod k$ and $h$, respectively. Then

$$
s_{1}\left(\left(\chi_{1}, k\right),\left(\chi_{2}, h\right)\right)+s_{1}\left(\left(\chi_{2}, h\right),\left(\chi_{1}, k\right)\right)=\frac{1}{u h k} \cdot \frac{B_{2, \chi_{1} \chi_{2}}}{2}+\frac{1}{u} B_{1, \chi_{1} \chi_{2}}+\Lambda,
$$

where $u=\chi_{1}(h) \chi_{2}(k)$ and $\Lambda$ is defined in (3.6).

4. Reciprocity law of $r$-ple Dedekind sums. In this section we define our $r$-ple Dedekind sums and prove the reciprocity law for them. As we mentioned in Section 1, Halbritter has already defined sums of type (1.1) as multiple Dedekind sums in [11].

Let $r$ be a positive integer.

DEFINITION 4.1. Let $z_{1}, \ldots, z_{r-1}$ be non-zero complex numbers, and set $\widetilde{z}=\left(z_{1}, \ldots, z_{r-1}\right)$. For a positive integer $n$, the $r$-ple Dedekind sum $\left(\mathbf{S}_{r}\right)_{n}(k, h ; \widetilde{z})$ with parameters $k, h$ and $\widetilde{z}$ is defined by

$$
\left(\mathbf{S}_{r}\right)_{n}(k, h ; \widetilde{z})=\sum_{b=0}^{h-1} \frac{b}{h}{ }_{r} S_{n}^{\prime}\left(\left\{\frac{k}{h} b\right\} ;\left(1, \frac{z_{1}}{h}, \ldots, \frac{z_{r-1}}{h}\right)\right) .
$$

When $r=1$, from Remark 2.3(1) we have $\left(\mathbf{S}_{1}\right)_{n}(k, h)=s_{n}(k, h)$. 
The main theorem which gives the reciprocity law of our $r$-ple Dedekind sums is the following:

TheORem 4.2. Let $\widetilde{z}$ be as in Definition 4.1. For a positive integer $n$,

$$
\begin{aligned}
& h^{n-1}\left(\mathbf{S}_{r}\right)_{n}(k, h ; \widetilde{z})+k^{n-1}\left(\mathbf{S}_{r}\right)_{n}(h, k ; \widetilde{z}) \\
& \quad={ }_{r+1} S_{n}^{\prime}\left(0 ;\left(k, h, z_{1}, \ldots, z_{r-1}\right)\right) \\
& \quad-\frac{1}{k h}{ }_{r+1} S_{n}^{\prime}\left(1 ;\left(1,1, z_{1}, \ldots, z_{r-1}\right)\right)+{ }_{r} S_{n}^{\prime}\left(0 ;\left(1, z_{1}, \ldots, z_{r-1}\right)\right) .
\end{aligned}
$$

Proof. First we prove the following lemma.

Lemma 4.3. Let $\alpha, \omega_{1}, \ldots, \omega_{r}$ be complex numbers with positive real parts. Then for $i$ with $1 \leq i \leq r$,

$$
\begin{aligned}
\zeta_{r+1}\left(s ; \alpha+\omega_{i},\left(\omega_{1}, \ldots, \omega_{i-1}, \omega_{i}, \omega_{i}, \omega_{i+1}, \ldots, \omega_{r}\right)\right) \\
=\sum_{m_{1}, \ldots, m_{r}=0}^{\infty} \frac{m_{i}}{\left(\alpha+m_{1} \omega_{1}+\ldots+m_{r} \omega_{r}\right)^{s}}
\end{aligned}
$$

for $\operatorname{Re}(s)>r+1$.

Proof. From the definition of $\zeta_{r+1}$,

$$
\begin{aligned}
& \zeta_{r+1}\left(s ; \alpha+\omega_{i},\left(\omega_{1}, \ldots, \omega_{i-1}, \omega_{i}, \omega_{i}, \omega_{i+1}, \ldots, \omega_{r}\right)\right) \\
& =\sum_{m_{1}, \ldots, m_{i}^{\prime}, m_{i}^{\prime \prime}, \ldots, m_{r}=0}^{\infty} \frac{1}{\left(\alpha+\omega_{i}+m_{1} \omega_{1}+\ldots+m_{i}^{\prime} \omega_{i}+m_{i}^{\prime \prime} \omega_{i}+\ldots+m_{r} \omega_{r}\right)^{s}} \\
& =\sum_{m_{1}, \ldots, m_{i}^{\prime}, m_{i}^{\prime \prime}, \ldots, m_{r}=0}^{\infty} \frac{1}{\left(\alpha+m_{1} \omega_{1}+\ldots+\left(1+m_{i}^{\prime}+m_{i}^{\prime \prime}\right) \omega_{i}+\ldots+m_{r} \omega_{r}\right)^{s}} .
\end{aligned}
$$

Here we set $m_{i}=1+m_{i}^{\prime}+m_{i}^{\prime \prime}$. Then this is reduced to (4.2).

Now we begin to prove Theorem 4.2. We first assume that each $z_{i}$ has a positive real part, and use Barnes's $(r+1)$-ple zeta function

$$
\begin{aligned}
\widetilde{\zeta}_{r+1}\left(s ;\left(k, h, z_{1}, \ldots, z_{r-1}\right)\right) & \\
= & \sum_{\substack{m_{1}, \ldots, m_{r+1}=0 \\
\left(m_{1}, \ldots, m_{r+1}\right) \neq(0, \ldots, 0)}}^{\infty} \frac{1}{\left(m_{1} k+m_{2} h+m_{3} z_{1}+\ldots+m_{r+1} z_{r-1}\right)^{s}} .
\end{aligned}
$$

As in the proof of Theorem 3.1, we set $m_{1}=b+h m_{1}^{\prime}$ and $m_{2}=a+k m_{2}^{\prime}$, 
and let $a, b$ vary from 0 to $k-1$ and from 0 to $h-1$, respectively. Then

$$
\begin{aligned}
& \widetilde{\zeta}_{r+1}\left(s ;\left(k, h, z_{1}, \ldots, z_{r-1}\right)\right)=\sum_{a=0}^{k-1} \sum_{b=0}^{h-1} \\
& \sum_{m_{1}^{\prime}, m_{2}^{\prime}, m_{3}, \ldots, m_{r+1}=0}^{\infty} \frac{1}{\left(k b+h a+k h\left(m_{1}^{\prime}+m_{2}^{\prime}\right)+m_{3} z_{1}+\ldots+m_{r+1} z_{r-1}\right)^{s}},
\end{aligned}
$$

where $\sum^{\prime}$ means that we sum over all non-negative integers $m_{1}^{\prime}, m_{2}^{\prime}, m_{3}, \ldots$ $\ldots, m_{r+1}$ except for $\left(m_{1}^{\prime}, m_{2}^{\prime}, m_{3}, \ldots, m_{r+1}\right)=(0, \ldots, 0)$ when $a=b=0$. By setting $m_{1}^{\prime}+m_{2}^{\prime}=N$, we have

$$
\begin{aligned}
& \widetilde{\zeta}_{r+1}\left(s ;\left(k, h, z_{1}, \ldots, z_{r-1}\right)\right) \\
& =\sum_{a=0}^{k-1} \sum_{b=0}^{h-1} \sum_{N, m_{3}, \ldots, m_{r+1}=0}^{\infty} \frac{N+1}{\left(k b+h a+k h N+\ldots+m_{r+1} z_{r-1}\right)^{s}} \\
& =\sum_{a=0}^{k-1} \sum_{b=0}^{h-1} \sum_{N, m_{3}, \ldots, m_{r+1}=0}^{\infty}\left\{\frac{1}{(k h)^{s}\left(\frac{k b+h a}{k h}+N+\ldots+m_{r+1} \frac{z_{r-1}}{k h}\right)^{s-1}}\right. \\
& +\frac{1}{(k h)^{s}\left(\frac{k b+h a}{k h}+N+\ldots+m_{r+1} \frac{z_{r-1}}{k h}\right)^{s}} \\
& -\frac{k b+h a}{(k h)^{s+1}} \cdot \frac{1}{\left(\frac{k b+h a}{k h}+N+\ldots+m_{r+1} \frac{z_{r-1}}{k h}\right)^{s}} \\
& \left.-\sum_{i=1}^{r-1} \frac{z_{i}}{(k h)^{s+1}} \cdot \frac{m_{i+2}}{\left(\frac{k b+h a}{k h}+N+\ldots+m_{r+1} \frac{z_{r-1}}{k h}\right)^{s}}\right\} \\
& =\frac{1}{(k h)^{s}} \sum_{a=0}^{k-1} \sum_{b=0}^{h-1} \zeta_{r}^{*}\left(s-1 ; \frac{k b+h a}{k h},\left(1, \frac{z_{1}}{k h}, \ldots, \frac{z_{r-1}}{k h}\right)\right) \\
& +\frac{1}{(k h)^{s}} \sum_{a=0}^{k-1} \sum_{b=0}^{h-1} \zeta_{r}^{*}\left(s ; \frac{k b+h a}{k h},\left(1, \frac{z_{1}}{k h}, \ldots, \frac{z_{r-1}}{k h}\right)\right) \\
& -\frac{1}{(k h)^{s}} \sum_{a=0}^{k-1} \sum_{b=0}^{h-1}\left(\frac{k b+h a}{k h}\right) \zeta_{r}^{*}\left(s ; \frac{k b+h a}{k h},\left(1, \frac{z_{1}}{k h}, \ldots, \frac{z_{r-1}}{k h}\right)\right) \\
& -\frac{1}{(k h)^{s}} \sum_{a=0}^{k-1} \sum_{b=0}^{h-1} \sum_{i=1}^{r-1} \frac{z_{i}}{k h} \\
& \times \zeta_{r+1}\left(s ; \frac{k b+h a+z_{i}}{k h},\left(1, \frac{z_{1}}{k h}, \ldots, \frac{z_{i}}{k h}, \frac{z_{i}}{k h}, \ldots, \frac{z_{r-1}}{k h}\right)\right)
\end{aligned}
$$

from Lemma 4.3, where $\zeta_{r}^{*}$ is the function defined in (2.9). 
We set $s=1-n$ for a positive integer $n$. Then by (2.5) and (2.7), we obtain

$$
\begin{aligned}
& \widetilde{\zeta}_{r+1}\left(1-n ;\left(k, h, z_{1}, \ldots, z_{r-1}\right)\right) \\
& =\frac{1}{(k h)^{1-n}} \sum_{a=0}^{k-1} \sum_{b=0}^{h-1} \frac{(-1)^{r}{ }_{r} S_{n+1}^{\prime}\left(\frac{k b+h a}{k h} ;\left(1, \frac{z_{1}}{k h}, \ldots, \frac{z_{r-1}}{k h}\right)\right)}{n+1} \\
& +\frac{1}{(k h)^{1-n}}\left\{\sum_{a=0}^{k-1} \sum_{b=0}^{h-1} \frac{(-1)^{r}{ }_{r} S_{n}^{\prime}\left(\frac{k b+h a}{k h} ;\left(1, \frac{z_{1}}{k h}, \ldots, \frac{z_{r-1}}{k h}\right)\right)}{n}-\delta\right\} \\
& -\frac{1}{(k h)^{1-n}} \sum_{a=0}^{k-1} \sum_{b=0}^{h-1}\left(\frac{a}{k}+\frac{b}{h}\right) \cdot \frac{(-1)^{r}{ }_{r} S_{n}^{\prime}\left(\frac{k b+h a}{k h} ;\left(1, \frac{z_{1}}{k h}, \ldots, \frac{z_{r-1}}{k h}\right)\right)}{n} \\
& -\frac{1}{(k h)^{1-n}} \sum_{a=0}^{k-1} \sum_{b=0}^{h-1} \sum_{i=1}^{r-1} \frac{z_{i}}{k h} \\
& \times \frac{(-1)^{r+1}{ }_{r+1} S_{n}^{\prime}\left(\frac{k b+h a}{k h}+\frac{z_{i}}{k h} ;\left(1, \frac{z_{1}}{k h}, \ldots, \frac{z_{i}}{k h}, \frac{z_{i}}{k h}, \ldots, \frac{z_{r-1}}{k h}\right)\right)}{n},
\end{aligned}
$$

where $\delta=1$ when $n=1$ and $\delta=0$ otherwise.

Before proceeding with the proof, several lemmas are needed.

Lemma 4.4. Let $x, \omega_{1}, \ldots, \omega_{r-1}$ be complex numbers with each $\omega_{i} \neq 0$. Then the following difference equation holds:

$$
\begin{aligned}
{ }_{r} S_{n}^{\prime}(x+1 ; & \left.\left(1, \omega_{1}, \ldots, \omega_{r-1}\right)\right) \\
& ={ }_{r} S_{n}^{\prime}\left(x ;\left(1, \omega_{1}, \ldots, \omega_{r-1}\right)\right)+{ }_{r-1} S_{n}^{\prime}\left(x ;\left(\omega_{1}, \ldots, \omega_{r-1}\right)\right) .
\end{aligned}
$$

Proof. This is shown by the following identities:

$$
\begin{aligned}
\frac{(-1)^{r} t e^{-(x+1) t}}{\left(1-e^{-t}\right) \ldots\left(1-e^{-\omega_{r-1} t}\right)}-\frac{(-1)^{r} t e^{-x t}}{\left(1-e^{-t}\right) \ldots\left(1-e^{-\omega_{r-1} t}\right)} \\
=\frac{(-1)^{r-1} t e^{-x t}}{\left(1-e^{-\omega_{1} t}\right) \ldots\left(1-e^{-\omega_{r-1} t}\right)} .
\end{aligned}
$$

From Definition 2.2 , by comparing the coefficients of $t^{n}$ above, we obtain (4.4).

Lemma 4.5. Let $x, \omega_{1}, \ldots, \omega_{r-1}$ be as in Lemma 4.4 and $N$ be a natural number. Then 


$$
\begin{aligned}
& \begin{array}{l}
\sum_{i=0}^{N-1}{ }_{r} S_{n}^{\prime}\left(x+\frac{i}{N} ;\left(1, \omega_{1}, \ldots, \omega_{r-1}\right)\right) \\
\quad=N^{1-n}{ }_{r} S_{n}^{\prime}\left(N x ;\left(1, N \omega_{1}, \ldots, N \omega_{r-1}\right)\right), \\
\sum_{i=0}^{N-1}{ }_{r} S_{n}^{\prime}\left(\left\{x+\frac{i}{N}\right\} ;\left(1, \omega_{1}, \ldots, \omega_{r-1}\right)\right) \\
=N^{1-n}{ }_{r} S_{n}^{\prime}\left(\{N x\} ;\left(1, N \omega_{1}, \ldots, N \omega_{r-1}\right)\right) .
\end{array}
\end{aligned}
$$

Proof. (4.5) can be shown from the generating function.

As for (4.6), since both sides have period $1 / N$, it suffices to prove it for $0 \leq x<1 / N$. But then it is just (4.5).

Lemma 4.6. Let $A, \omega_{1}, \ldots, \omega_{r}$ be complex numbers with each $\omega_{i} \neq 0$, and set $\widetilde{\omega}=\left(\omega_{1}, \ldots, \omega_{r}\right)$. Then

$$
\begin{aligned}
\sum_{i=1}^{r} \omega_{i} \cdot{ }_{r+1} S_{n}^{\prime}\left(A+\omega_{i} ;\left(\omega_{1}, \ldots, \omega_{i}, \omega_{i}, \omega_{i+1}, \ldots, \omega_{r}\right)\right) \\
=A \cdot{ }_{r} S_{n}^{\prime}(A ; \widetilde{\omega})-\frac{n}{n+1}{ }_{r} S_{n+1}^{\prime}(A ; \widetilde{\omega}) .
\end{aligned}
$$

Proof. We compute the derivative of $\frac{(-1)^{r} t e^{-A t}}{\left(1-e^{-\omega_{1} t}\right) \ldots\left(1-e^{-\omega_{r} t}\right)}$ with respect to $t$. Then

$$
\begin{aligned}
\frac{d}{d t}( & \left.\frac{(-1)^{r} t e^{-A t}}{\left(1-e^{-\omega_{1} t}\right) \ldots\left(1-e^{-\omega_{r} t}\right)}\right) \\
= & \frac{1}{t} \cdot \frac{(-1)^{r} t e^{-A t}}{\left(1-e^{-\omega_{1} t}\right) \ldots\left(1-e^{-\omega_{r} t}\right)}-A \frac{(-1)^{r} t e^{-A t}}{\left(1-e^{-\omega_{1} t}\right) \ldots\left(1-e^{-\omega_{r} t}\right)} \\
& \quad+\sum_{i=1}^{r} \frac{(-1)^{r+1} \omega_{i} t e^{-\left(A+\omega_{i}\right) t}}{\left(1-e^{-\omega_{1} t}\right) \ldots\left(1-e^{-\omega_{i} t}\right)^{2}\left(1-e^{-\omega_{i+1} t}\right) \ldots\left(1-e^{-\omega_{r} t}\right)} .
\end{aligned}
$$

Here

$$
\begin{aligned}
& \frac{d}{d t}\left(\frac{(-1)^{r} t e^{-A t}}{\left(1-e^{-\omega_{1} t}\right) \ldots\left(1-e^{-\omega_{r} t}\right)}\right) \\
& \quad=\sum_{k=2}^{r} \frac{(-1)^{k-1}(k-1) A_{k}(A ; \widetilde{\omega})}{t^{k}}+\sum_{n=1}^{\infty} \frac{(-1)^{n-1}{ }_{r} S_{n}^{\prime}(A ; \widetilde{\omega})}{(n-1) !} t^{n-1}
\end{aligned}
$$

By comparing the coefficients of $t^{n}$ in (4.8) and (4.9), we obtain (4.7).

We now return to the proof of Theorem 4.2. Let $S$ denote the set as in (3.3), i.e. $S=\left\{(a, b) \in \mathbb{Z}^{2} \mid 0 \leq a<k, 0 \leq b<h, a / k+b / h>1\right\}$. By (4.4) and (4.6), 


$$
\begin{aligned}
\sum_{a=0}^{k-1} \sum_{b=0}^{h-1} \frac{b}{h}{ }_{r} S_{n}^{\prime}\left(\frac{k b+h a}{k h} ;\left(1, \frac{z_{1}}{k h}, \ldots, \frac{z_{r-1}}{k h}\right)\right) \\
=\sum_{b=0}^{h-1} \frac{b}{h} \sum_{a=0}^{k-1}{ }_{r} S_{n}^{\prime}\left(\left\{\frac{k b+h a}{k h}\right\} ;\left(1, \frac{z_{1}}{k h}, \ldots, \frac{z_{r-1}}{k h}\right)\right) \\
+\sum_{(a, b) \in S} \frac{b}{h}{ }^{r-1} S_{n}^{\prime}\left(\left\{\frac{k b+h a}{k h}\right\} ;\left(\frac{z_{1}}{k h}, \ldots, \frac{z_{r-1}}{k h}\right)\right) \\
=k^{1-n}\left(\mathbf{S}_{r}\right)_{n}(k, h ; \widetilde{z}) \\
+\sum_{(a, b) \in S} \frac{b}{h}_{r-1} S_{n}^{\prime}\left(\left\{\frac{k b+h a}{k h}\right\} ;\left(\frac{z_{1}}{k h}, \ldots, \frac{z_{r-1}}{k h}\right)\right) .
\end{aligned}
$$

Similarly we have

$$
\begin{aligned}
& \sum_{a=0}^{k-1} \sum_{b=0}^{h-1} \frac{a}{k}{ }_{r} S_{n}^{\prime}\left(\frac{k b+h a}{k h} ;\left(1, \frac{z_{1}}{k h}, \ldots, \frac{z_{r-1}}{k h}\right)\right) \\
= & h^{1-n}\left(\mathbf{S}_{r}\right)_{n}(h, k ; \widetilde{z})+\sum_{(a, b) \in S} \frac{a}{k}{ }_{r-1} S_{n}^{\prime}\left(\left\{\frac{k b+h a}{k h}\right\} ;\left(\frac{z_{1}}{k h}, \ldots, \frac{z_{r-1}}{k h}\right)\right) .
\end{aligned}
$$

Also by (4.4) and (4.5), for $\alpha, \omega_{1}, \ldots, \omega_{r-1}$ with each $\omega_{i} \neq 0$,

$$
\begin{aligned}
\sum_{a=0}^{k-1} \sum_{b=0}^{h-1}{ }_{r} S_{n}^{\prime}\left(\frac{k b+h a}{k h}+\frac{\alpha}{k h} ;\left(1, \frac{\omega_{1}}{k h}, \ldots, \frac{\omega_{r-1}}{k h}\right)\right) \\
=\sum_{(a, b) \notin S}{ }_{r} S_{n}^{\prime}\left(\left\{\frac{k b+h a}{k h}\right\}+\frac{\alpha}{k h} ;\left(1, \frac{\omega_{1}}{k h}, \ldots, \frac{\omega_{r-1}}{k h}\right)\right) \\
+\sum_{(a, b) \in S}{ }_{r} S_{n}^{\prime}\left(\left\{\frac{k b+h a}{k h}\right\}+\frac{\alpha}{k h}+1 ;\left(1, \frac{\omega_{1}}{k h}, \ldots, \frac{\omega_{r-1}}{k h}\right)\right) \\
=\sum_{a=0}^{k-1} \sum_{b=0}^{h-1}{ }_{r} S_{n}^{\prime}\left(\left\{\frac{k b+h a}{k h}\right\}+\frac{\alpha}{k h} ;\left(1, \frac{\omega_{1}}{k h}, \ldots, \frac{\omega_{r-1}}{k h}\right)\right) \\
+\sum_{(a, b) \in S}{ }_{r-1} S_{n}^{\prime}\left(\left\{\frac{k b+h a}{k h}\right\}+\frac{\alpha}{k h} ;\left(\frac{\omega_{1}}{k h}, \ldots, \frac{\omega_{r-1}}{k h}\right)\right) \\
=(k h)^{1-n}{ }_{r} S_{n}^{\prime}\left(\alpha ;\left(1, \omega_{1}, \ldots, \omega_{r-1}\right)\right) \\
+\sum_{(a, b) \in S}{ }_{r-1} S_{n}^{\prime}\left(\left\{\frac{k b+h a}{k h}\right\}+\frac{\alpha}{k h} ;\left(\frac{\omega_{1}}{k h}, \ldots, \frac{\omega_{r-1}}{k h}\right)\right)
\end{aligned}
$$


since

$$
\begin{aligned}
\left\{\left\{\frac{k b+h a}{k h}\right\} \mid 0 \leq a<k, 0 \leq b<h, a, b \in \mathbb{Z}\right\} & \\
& =\left\{\frac{i}{k h} \mid 0 \leq i \leq k h-1\right\} .
\end{aligned}
$$

By using (4.12) three times and also by (4.10) and (4.11), the identity (4.3) becomes

$$
\begin{aligned}
& \widetilde{\zeta}_{r+1}\left(1-n ;\left(k, h, z_{1}, \ldots, z_{r-1}\right)\right) \\
& =\frac{(-1)^{r}}{(k h)^{1-n}(n+1)}\left\{(k h)^{-n}{ }_{r} S_{n+1}^{\prime}\left(0 ;\left(1, z_{1}, \ldots, z_{r-1}\right)\right)\right. \\
& \left.+\sum_{(a, b) \in S}{ }_{r-1}^{\prime} S_{n+1}^{\prime}\left(\left\{\frac{k b+h a}{k h}\right\} ;\left(\frac{z_{1}}{k h}, \ldots, \frac{z_{r-1}}{k h}\right)\right)\right\} \\
& +\frac{(-1)^{r}}{(k h)^{1-n} n}\left\{(k h)^{1-n}{ }_{r} S_{n}^{\prime}\left(0 ;\left(1, z_{1}, \ldots, z_{r-1}\right)\right)\right. \\
& \left.+\sum_{(a, b) \in S}{ }_{r-1} S_{n}^{\prime}\left(\left\{\frac{k b+h a}{k h}\right\} ;\left(\frac{z_{1}}{k h}, \ldots, \frac{z_{r-1}}{k h}\right)\right)\right\} \\
& -\delta-\frac{(-1)^{r}}{(k h)^{1-n} n}\left\{k^{1-n}\left(\mathbf{S}_{r}\right)_{n}(k, h ; \widetilde{z})\right. \\
& +\sum_{(a, b) \in S} \frac{b}{h} r-1 S_{n}^{\prime}\left(\left\{\frac{k b+h a}{k h}\right\} ;\left(\frac{z_{1}}{k h}, \ldots, \frac{z_{r-1}}{k h}\right)\right) \\
& +h^{1-n}\left(\mathbf{S}_{r}\right)_{n}(h, k ; \widetilde{z}) \\
& \left.+\sum_{(a, b) \in S} \frac{a}{k} r-1 S_{n}^{\prime}\left(\left\{\frac{k b+h a}{k h}\right\} ;\left(\frac{z_{1}}{k h}, \ldots, \frac{z_{r-1}}{k h}\right)\right)\right\} \\
& +\frac{(-1)^{r}}{(k h)^{1-n} n}\left[\sum _ { i = 1 } ^ { r - 1 } \frac { z _ { i } } { k h } \left\{(k h)_{r+1}^{1-n} S_{n}^{\prime}\left(z_{i} ;\left(1, z_{1}, \ldots, z_{i}, z_{i}, \ldots, z_{r-1}\right)\right)\right.\right. \\
& \left.\left.+\sum_{(a, b) \in S} S_{n}^{\prime}\left(\left\{\frac{k b+h a}{k h}\right\}+\frac{z_{i}}{k h} ;\left(\frac{z_{1}}{k h}, \ldots, \frac{z_{i}}{k h}, \frac{z_{i}}{k h}, \ldots, \frac{z_{r-1}}{k h}\right)\right)\right\}\right] .
\end{aligned}
$$

Now we use Lemma 4.6. By replacing $r$ with $r-1$ and taking $\left\{\frac{k b+h a}{k h}\right\}$ as $A$ and $z_{i} /(k h)$ as $\omega_{i}$ in (4.7), we have 


$$
\begin{aligned}
\sum_{i=1}^{r-1} \frac{z_{i}}{k h} \cdot{ }_{r} S_{n}^{\prime}\left(\left\{\frac{k b+h a}{k h}\right\}+\frac{z_{i}}{k h} ;\left(\frac{z_{1}}{k h}, \ldots, \frac{z_{i}}{k h}, \frac{z_{i}}{k h}, \ldots, \frac{z_{r-1}}{k h}\right)\right) \\
=\left\{\frac{k b+h a}{k h}\right\}_{r-1} S_{n}^{\prime}\left(\left\{\frac{k b+h a}{k h}\right\} ;\left(\frac{z_{1}}{k h}, \ldots, \frac{z_{r-1}}{k h}\right)\right) \\
-\frac{n}{n+1}{ }_{r-1} S_{n+1}^{\prime}\left(\left\{\frac{k b+h a}{k h}\right\} ;\left(\frac{z_{1}}{k h}, \ldots, \frac{z_{r-1}}{k h}\right)\right) .
\end{aligned}
$$

From this the sum of $\sum_{(a, b) \in S}$ in (4.13) becomes 0 . Also by taking 0 as $A$, 1 as $\omega_{1}$ and $z_{i}$ as $\omega_{i+1}$ in (4.7), we have

$$
\begin{aligned}
r+1 & S_{n}^{\prime}\left(1 ;\left(1,1, z_{1}, \ldots, z_{r-1}\right)\right)+\sum_{i=1}^{r-1} z_{i} \cdot \\
& r+1 S_{n}^{\prime}\left(z_{i} ;\left(1, z_{1}, \ldots, z_{i}, z_{i}, \ldots, z_{r-1}\right)\right) \\
& =-\frac{n}{n+1} r S_{n+1}^{\prime}\left(0 ;\left(1, z_{1}, \ldots, z_{r-1}\right)\right) .
\end{aligned}
$$

Substituting this into (4.13), we obtain

$$
\begin{aligned}
\widetilde{\zeta}_{r+1}\left(1-n ;\left(k, h, z_{1}, \ldots, z_{r-1}\right)\right)= & \frac{(-1)^{r}}{n}{ }_{r} S_{n}^{\prime}\left(0 ;\left(1, z_{1}, \ldots, z_{r-1}\right)\right)-\delta \\
& -\frac{(-1)^{r}}{n} h^{n-1}\left(\mathbf{S}_{r}\right)_{n}(k, h ; \widetilde{z}) \\
& -\frac{(-1)^{r}}{n} k^{n-1}\left(\mathbf{S}_{r}\right)_{n}(h, k ; \widetilde{z}) \\
& -\frac{(-1)^{r}}{k h n} r+1 S_{n}^{\prime}\left(1 ;\left(1,1, z_{1}, \ldots, z_{r-1}\right)\right) .
\end{aligned}
$$

On the other hand, by (2.7),

$$
\widetilde{\zeta}_{r+1}\left(1-n ;\left(k, h, z_{1}, \ldots, z_{r-1}\right)\right)=\frac{(-1)^{r+1}{ }_{r+1} S_{n}^{\prime}\left(0 ;\left(k, h, z_{1}, \ldots, z_{r-1}\right)\right)}{n}-\delta,
$$

and thus the law (4.1) is obtained when each $z_{i}$ has a positive real part.

Since both sides of (4.1) are rational functions in $z_{1}, \ldots, z_{r-1}$ with only $z_{1} \ldots z_{r-1}$ in the denominator, the law is valid for any $\widetilde{z}$ with each $z_{i} \neq 0$. This completes the proof of Theorem 4.2.

REMARK 4.7. When $r=1$, Theorem 4.2 coincides with Apostol's reciprocity law (Theorem A). This is because ${ }_{2} S_{n}^{\prime}(0 ;(k, h))=n \cdot \widetilde{\zeta}_{2}(1-n ;(k, h))$ $+\delta$ and

$$
{ }_{2} S_{n}^{\prime}(1 ;(1,1))=-\frac{n}{n+1}{ }_{1} S_{n+1}^{\prime}(0 ;(1))=-\frac{n}{n+1} B_{n+1}
$$

by (4.7). 
Acknowledgements. The authors thank an anonymous referee for comments on an earlier version of this paper. They also thank Kohji Matsumoto for pointing out the work of Egami ([9]).

\section{References}

[1] T. Apostol, Generalized Dedekind sums and the transformation formulae of certain Lambert series, Duke Math. J. 17 (1950), 147-157.

[2] E. W. Barnes, The theory of the double gamma function, Philos. Trans. Roy. Soc. Ser. A 196 (1901), 265-387.

[3] - On the theory of the multiple gamma function, Trans. Cambridge Philos. Soc. 19 (1904), 374-425.

[4] B. C. Berndt, Character transformation formulae similar to those for the Dedekind eta-function, in: Proc. Sympos. Pure Math. 24, Amer. Math. Soc., Providence, RI, 1973, 9-30.

[5] -, On Eisenstein series with characters and the values of Dirichlet L-functions, Acta Arith. 28 (1975), 299-320.

[6] -, Reciprocity theorems for Dedekind sums and generalizations, Adv. Math. 23 (1977), 285-316.

[7] - Analytic Eisenstein series, theta-functions and series relations in the spirit of Ramanujan, J. Reine Angew. Math. 303/304 (1978), 332-365.

[8] R. Chapman, Reciprocity laws for generalized higher dimensional Dedekind sums, Acta Arith. 93 (2000), 189-199.

[9] S. Egami, Reciprocity laws of multiple zeta functions and generalized Dedekind sums, in: Analytic Number Theory and Related Topics, K. Nagasaka (ed.), World Sci., 1993, 17-27.

[10] U. Halbritter, Berechnung der Werte von Zetafunktionen totalreeller kubischer Zahlkörper an ganzzahligen Stellen mittels verallgemeinerter Dedekindscher Summen, I, J. Reine Angew. Math. 361 (1985), 95-117.

[11] —, Berechnung der Werte von Zetafunktionen totalreeller kubischer Zahlkörper an ganzzahligen Stellen mittels verallgemeinerter Dedekindscher Summen, II, Results in Math. 13 (1988), 99-134.

[12] R. R. Hall, J. C. Wilson and D. Zagier, Reciprocity formulae for general DedekindRademacher sums, Acta Arith. 73 (1995), 389-397.

[13] S. Hu, Shintani cocycles and generalized Dedekind sums, Ph.D. thesis, Univ. of Pennsylvania, 1997.

[14] S. Hu and D. Solomon, Properties of higher-dimensional Shintani generating functions and cocycles on $P G L_{3}(\mathbf{Q})$, Proc. London Math. Soc. (3) 82 (2001), $64-88$.

[15] S. Lang, Cyclotomic Fields, Grad. Texts in Math. 59, Springer, 1978.

[16] K. Ota, Derivatives of Dedekind sums and their reciprocity law, J. Number Theory, to appear.

[17] - Dedekind sums with characters and class numbers of imaginary quadratic fields, preprint, 2001.

[18] H. Rademacher, Topics in Analytic Number Theory, Grundlehren Math. Wiss. 169, Springer, 1973.

[19] C. L. Siegel, Bernoullische Polynome und quadratische Zahlenkörper, Nachr. Akad. Wiss. Göttingen Math.-Phys. Kl. II 1968, 7-38. 
[20] D. Solomon, Algebraic properties of Shintani's generating functions: Dedekind sums and cocycles on $P G L_{2}(\mathbf{Q})$, Compositio Math. 112 (1998), 333-362.

[21] D. Zagier, Higher dimensional Dedekind sums, Math. Ann. 202 (1973), 149-172.

Department of Mathematics

and Computer Science

Tsuda College

Kodaira-shi, Tokyo 187-8577, Japan

E-mail: ota@tsuda.ac.jp

Received on 3.1.2001

and in revised form on 24.6.2002 